Pacific Journal of Mathematics

A CHARACTERIZATION OF UNIQUELY DIVISIBLE
COMMUTATE SEMIGROUPS 


\title{
A CHARACTERIZATION OF UNIQUELY DIVISIBI.E COMMUTATIVE SEMIGROUPS
}

\author{
D. R. BRown AND J. G. LATORRE
}

Let $(S,+)$ be a commutative semigroup. If, for each $x \in S$, and for each positive integer $n$, there exists an (unique) element $y$ of $S$ such that $x=n y$, then $S$ is (uniquely) divisible. In this note we present a more or less intrinsic characterization of uniquely divisible commutative semigroups and remark on a special sub-class of these semigroups in which it is possible to discern the fine structure of the addition.

2. The characterization. Let $P$ represent the additive semigroup of positive rational numbers. By a cone of a rational vector space we mean a convex subset $C$ such that $P C \subset C$ and $-P C \cap C=0$. A commutative semigroup is separative if $2 a=a+b=2 b$ implies $a=b$ for any $a, b \in S$. Let $L$ be the maximal (lower) semilattice homomorphic image of $S$, and let $h$ be the natural map of $S$ onto $L$. For $e \in L$, let $h^{-1}(e)=S_{e}$. The Hewitt-Zuckerman theorem [3; or 1, Th. 4.18] states that, if $S$ is separative, then each $S_{e}$ is cancellative, and $S$ is isomorphically embeddable in a semilattice of groups, $\left\{V_{e}\right\}$ in such a way that each $V_{e}$ is the difference group of $S_{e}$, and the semilattice is isomorphic to $L$.

Since an uniquely divisible commutative semigroup is clearly separative, we have immediately that any such entity is isomorphic to a divisible subsemigroup of a semilattice of divisible groups. Indeed, each $V_{e}$ must be uniquely divisible, and hence a rational vector space (see [4], for example). Furthermore, since each $S_{e}$ is cancellative, it follows from Hancock's theorem [2, Th. 7] that each $S_{e}$ is the direct sum of a rational vector space and a cone of a rational vector space. We have now:

THEOREM 1. Let $S$ be an uniquely divisible commutative semigroup. Then $S$ is a semilattice of subsemigroups $S_{e}$, each of which is the direct sum of a rational vector space and a cone of a rational vector space. Furthermore, the addition in $S$ is determined by semigroup homomorphisms between these subsemigroups which are restrictions of homomorphisms (linear maps) between their difference groups.

3. A special case. We now restrict our attention to the situation in which, for each $e \in L, S_{e} \cong P$. In this case, any $x_{e} \in S_{e}$ satisfies 
$P x_{e}=S_{e}$. By $x_{\alpha}$ we shall mean an element of $S_{\alpha}$.

Lemma 1. Let $e, f \in L, e \leqq f$; let $x_{e}+x_{f}=r x_{e}, r \in P . \quad$ Then $r \geqq 1$, and for $s, t \in P, s x_{e}+t x_{f}=[s+t(r-1)] x_{e}$.

Proof. Suppose $r<1$ and let $z=x_{e}+(1 /(1-r)) x_{f}$. Then

$$
z=\left[\left(x_{e}+x_{f}\right)+\left(\frac{r}{1-r}\right) x_{f}\right]=\left[r x_{e}+\left(\frac{r}{1-r}\right) x_{f}\right]=r z .
$$

Hence, $r=1$, which is a contradiction.

Now, consider $S$ as embedded in a semilattice of rational vector spaces as in the proof of Theorem 1 . We have

$$
\begin{aligned}
s x_{e}+t x_{f} & =\left(s x_{e}+0_{e}\right)+t x_{f} \\
& =s x_{e}+\left(0_{e}+t x_{f}\right) \\
& =s x_{e}+t\left(0_{e}+x_{f}\right) \\
& =s x_{e}+t\left([r-1] x_{e}\right) \\
& =(s+t[r-1]) x_{e}
\end{aligned}
$$

The proof is now complete.

LEMma 2. Let $e, f, g \in L, e \leqq f \leqq g$. Suppose $x_{e}+x_{f}=a x_{e}, x_{e}+$ $x_{g}=b x_{e}, x_{f}+x_{g}=c x_{f}, a, b, c \in P$. If any two of $a, b, c$ equal 2 , then $a=b=c=2$.

Proof. Note $[a+(b-1)] x_{e}=a x_{e}+x_{g}=\left(x_{e}+x_{f}\right)+x_{g}=x_{e}+\left(x_{f}+x_{g}\right)=$ $x_{e}+c x_{f}=[1+c(a-1)] x_{e}$. By the uniqueness of roots, $a+b-1=$ $1+c(a-1)$, and proof is complete.

Lemma 3. Let $e, f \in L$. If $x_{e}+x_{e f}=x_{f}+x_{e f}=2 x_{e f}$, then $x_{e}+$ $x_{f}=2 x_{e f}$.

Proof. Let $x_{e}+x_{f}=a x_{e f}$. Then $3 x_{e f}=x_{e f}+\left(x_{e f}+x_{e}\right)=2 x_{e f}+x_{e}=$ $\left(x_{e f}+x_{f}\right)+x_{e}=(1+a) x_{e f}$. Hence $a=2$.

THEOREM 2. Let $S$ be an uniquely divisible commutative semigroup such that $x+y \neq y$, all $x, y \in S$. Then $S \cong P \times L$.

Proof. Fix $e \in L, x_{e} \in S_{e}$. For each $f \in L$, choose $x_{f} \in S_{f}$ such that:

(1) $x_{e}+x_{f}=2 x_{f}$ if $f \leqq e$,

(2) $x_{f}+x_{e f}=2 x_{e f}$ otherwise.

Lemma 1 assures the availability of such elements; there is no 
ambiguity involved provided (1) is accomplished before (2). Fix $f, g \in L$; we shall show $x_{f}+x_{f g}=x_{g}+x_{f g}=2 x_{f g}$. To this end, note that $x_{e}+x_{e f}=2 x_{e f}$ and $x_{e}+x_{e f g}=2 x_{e f g}$ by (1) above. Hence, by Lemma $2, x_{e f}+x_{e f g}=2 x_{e f g}$. Since $x_{e}+x_{f}=x_{e f}$, we have $x_{f}+2 x_{e f}=3 x_{e f}$; by cancellation in $S_{e f}$, it follows that $x_{f}+x_{e f}=2 x_{e f}$. By applying Lemma 2 again, we have $x_{f}+x_{e f g}=2 x_{e f g}$. By an argument identical to the one involving $f$ and $e f$ above, $x_{f g}+x_{e f g}=2 x_{e f g}$. Finally, applying Lemma 2 for the final time, we have $x_{f}+x_{f g}=2 x_{f g}$. Similarly, $x_{g}+x_{f g}=2 x_{f g}$; by Lemma 3 it follows that $x_{f}+x_{g}=2 x_{f g}$. Finally, if, say $s \geqq t$, then $s x_{f}+t x_{g}=t\left(x_{f}+x_{g}\right)+(s-t) x_{f}=2 t x_{f g}+(s-t) x_{f}=$ $(s+t) x_{f g}$ by Lemma 1 . The function $\phi: S \rightarrow P \times L$ defined by $\phi\left(r x_{f}\right)=$ $(r, f)$ is now clearly an isomorphism.

Next, let $L$ be any semilattice, and let $\phi$ be a homomorphism of $L$ onto a chain $B$. For each $\beta \in B$, let $L_{\beta}=\phi^{-1}(\beta)$. For each $\beta$, let $S_{\beta}=P \times L_{\beta}$, and let $S=\cup\left\{S_{\beta}: \beta \in B\right\}$. Define an addition in $S$ by

$$
(r, e)+(s, f)=\left\{\begin{array}{l}
(r+s, e f) \text { if } e, f \in L_{\beta}, \\
(r, e f) \text { if } e \in L_{\beta}, f \in L_{\gamma}, \beta<\gamma, \\
(s, e f) \text { if } e \in L_{\beta}, f \in L_{\gamma}, \gamma<\beta .
\end{array}\right.
$$

With this addition, $S$ is an uniquely divisible commutative semigroup with maximal semilattice image $L$ and with each $S_{e} \cong P$. The class of semigroups thus defined will be referred to as being of type $\mathscr{E}$.

THEOREM 3. Let $S$ be an uniquely divisible commutative semigroup such that each $S_{e}$ is isomorphic to $P$. Then $S$ is isomorphic to a semigroup of type $\mathscr{E}$.

Proof. Define a relation $\sim$ on $S$ by $x \sim y$ if and only if $x+$ $(x+y) \neq x+y \neq y+(x+y)$. To check transitivity, let $x \sim y, y \sim z$. In particular, let $x+(x+y)=r(x+y), y+(y+z)=s(y+z)$, with $r, s>1$. Then $x+(x+y+z)=r(x+y)+z=r x+(r-1) y+(y+z)=$ $r x+[1+(r-1)(s-1)](y+z) \neq x+y+z$. Hence $x+(x+z) \neq x+z$. Similarly, $z+(x+z) \neq x+z$.

It follows by arguments similar to the above that $\sim$ is a congruence on $S$ and that $S / \sim$ is a chain. Let $j$ be the natural map of $S$ onto $S / \sim$; note that $j$ factors into the composition of $h$ and an induced map from $L$ to $S / \sim$. For $\beta \in S / \sim, j^{-1}(\beta)$ satisfies the conditions of Theorem 2. Specifically, $j^{-1}(\beta) \cong P \times h j^{-1}(\beta)$. Thus any $x \in j^{-1}(\beta)$ has an unique representation, $x=r x_{e}$, with $e \in h j^{-1}(\beta), r \in P$, and $x_{e}$ selected from $h^{-1}(e)$ in line with the proof of Theorem 2. Suppose $\beta, \gamma \in S / \sim \gamma, \beta<\gamma$, and let $r x_{e} \in j^{-1}(\beta), s x_{f} \in j^{-1}(\gamma)$. Then $x_{e}+x_{f} \in j^{-1}(\beta)$ and $x_{f}+\left(x_{e}+x_{f}\right)=x_{e}+x_{f}$. Let $x_{e}+x_{f}=t x_{e f}$. By Lemma 1, $x_{f}+$ $x_{e f}=x_{e f} ;$ since $x_{e}, x_{e f} \in j^{-1}(\beta), x_{e}+x_{e f}=2 x_{e f}$. Hence $(1+t) x_{e f}=x_{e f}+$ 
$\left(x_{e}+x_{f}\right)=\left(x_{e f}+x_{e}\right)+x_{f}=2 x_{e f}+x_{f}=2 x_{e f} ;$ hence $t=1$. Now, if, say $r \leqq s$, then $r x_{e}+s x_{f}=r\left(x_{e}+x_{f}\right)+(s-r) x_{f}=r x_{e f}+(s-r) x_{f}=$ $r x_{e f}$ by Lemma 1. If, on the other hand, $s<r$, then $r x_{e}+s x_{f}=$ $s\left(x_{e}+x_{f}\right)+(r-s) x_{e}=s x_{e f}+(r-s) x_{e}=r x_{e f}$ by Lemma 1. We have now shown that the addition of $S$ satisfies:

$$
r x_{e}+s x_{f}=\left\{\begin{array}{l}
(r+s) x_{e f} \text { if } j h^{-1}(e)=j h^{-1}(f), \\
r x_{e f f} \text { if } j h^{-1}(e)<j h^{-1}(f), \\
s x_{e f} \text { if } j h^{-1}(f)<j h^{-1}(e) .
\end{array}\right.
$$

The mapping $r x_{e} \rightarrow(r, e)$ now establishes that $S$ is isomorphic to a semigroup of type $\mathscr{E}$.

In closing, we remark that the relations used in proving Theorems 2 and 3 can be reformulated in terms of the homomorphisms guaranteed by Theorem 1. In Theorem 3 in particular, if $e \leqq f$, then $x_{e} \sim x_{f}$ if and only if the addition homomorphism is an isomorphism. Furthermore, if $x_{e}$ and $x_{f}$ are not equivalent, then the addition homomorphism is the zero mapping.

It is a pleasure to record our obligation to Professor A. H. Clifford for several valuable comments.

\section{REFERENCES}

1. A. H. Clifford and G. B. Preston, The algebraic theory of semigroups, Vol. 1, AMS Survey No. 7, Providence, 1961.

2. V. R. Hancock, On complete semimodules, Proc. Amer. Math. Soc. 11 (1960), 71-76.

3. E. Hewitt and H. S. Zuckerman, The $\mathscr{L}_{1}$-algebra of a commutative semigroup, Trans. Amer. Math. Soc. 83 (1956), 70-97.

4. I. Kaplansky, Infinite abelian groups, University of Michigan Press, Ann Arbor, 1954.

5. J. G. LaTorre, On uniquely divisible semigroups, Master's Thesis, The University of Tennessee, Knoxville, 1964.

6. T. Tamura, Minimal commutative divisible semigroups, Bull. Amer. Math. Soc. 69 (1963), 713-716.

Received February 18, 1965.

The University of Tennessee 


\section{PACIFIC JOURNAL OF MATHEMATICS}

H. SAMELSON

Stanford University Stanford, California

R. M. BLUMENTHAL

University of Washington

Seattle, Washington 98105

\section{EDITORS}

\author{
*J. DugundJI \\ University of Southern California \\ Los Angeles, California 90007 \\ RICHARD ARENS \\ University of California \\ Los Angeles, California 90024
}

\section{ASSOCIATE EDITORS}

E. F. BeCKENBACH

B. H. NEUMANN

F. WOLF

K. YosidA

\section{SUPPORTING INSTITUTIONS}

UNIVERSITY OF BRITISH COLUMBIA

CALIFORNIA INSTITUTE OF TECHNOLOGY

UNIVERSITY OF CALIFORNIA

MONTANA STATE UNIVERSITY

UNIVERSITY OF NEVADA

NEW MEXICO STATE UNIVERSITY

OREGON STATE UNIVERSITY

UNIVERSITY OF OREGON

OSAKA UNIVERSITY

UNIVERSITY OF SOUTHERN CALIFORNIA
STANFORD UNIVERSITY

UNIVERSITY OF TOKYO

UNIVERSITY OF UTAH

WASHINGTON STATE UNIVERSITY

UNIVERSITY OF WASHINGTON

AMERICAN MATHEMATICAL SOCIETY CHEVRON RESEARCH CORPORATION TRW SYSTEMS

NAVAL ORDNANCE TEST STATION 


\section{Pacific Journal of Mathematics}

Vol. 18, No. 1

March, 1966

Edward Joseph Barbeau, Semi-algebras that are lower semi-lattices ...... 1

Steven Fredrick Bauman, The Klein group as an automorphism group

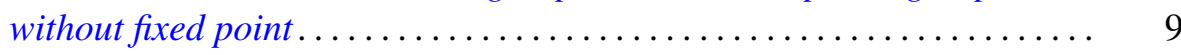

Homer Franklin Bechtell, Jr., Frattini subgroups and $\Phi$-central groups .... 15

Edward Kenneth Blum, A convergent gradient procedure in prehilbert

spaces ............................................

Edward Martin Bolger, The sum of two independent exponential-type random variables ...................................

David Wilson Bressler and A. P. Morse, Images of measurable sets .......

Dennison Robert Brown and J. G. LaTorre, A characterization of uniquely

divisible commutative semigroups........................

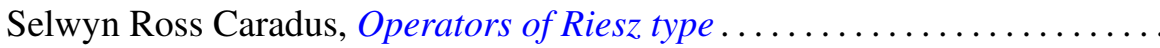

Jeffrey Davis and Isidore Isaac Hirschman, Jr., Toeplitz forms and ultraspherical polynomials ............................

Lorraine L. Foster, On the characteristic roots of the product of certain rational integral matrices of order two ......................

Alfred Gray and S. M. Shah, Asymptotic values of a holomorphic function

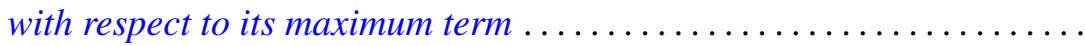

Sidney (Denny) L. Gulick, Commutativity and ideals in the biduals of

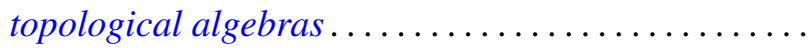

G. J. Kurowski, Further results in the theory of monodiffric functions

Lawrence S. Levy, Commutative rings whose homomorphic images are self-injective .

Calvin T. Long, On real numbers having normality of order $k$....

Bertram Mond, An inequality for operators in a Hilbert space. ...

John William Neuberger, The lack of self-adjointness in three-point boundary value problems ........................

C. A. Persinger, Subsets of $n$-books in $E^{3}$

Oscar S. Rothaus and John Griggs Thompson, A combinatorial problem in the symmetric group ............................... 175

Rodolfo DeSapio, Unknotting spheres via Smale .................. 179

James E. Shockley, On the functional equation

$$
F(m n) F((m, n))=F(m) F(n) f((m, n)) \ldots \ldots \ldots
$$

Kenneth Edward Whipple, Cauchy sequences in Moore spaces ... 